> Parce qu'elles représentent une réserve cellulaire quasi illimitée par leur propriété d'autorenouvellement et possèdent la capacité de se différencier dans tous les lignages cellulaires in vitro, les cellules souches embryonnaires, ou cellules $\varepsilon S$, constituent un outil de choix pour le développement de nouvelles approches thérapeutiques fondées sur le remplacement cellulaire. Chez la souris, l'analyse de la différenciation des cellules ES dans les lignées gliales et neuronales a été particulièrement bien étudiée, compte tenu de l'enjeu thérapeutique éventuel chez l'homme, mais aussi en raison de la propension naturelle de ces cellules à adopter ce destin cellulaire, et de la flexibilité de leur capacité de différenciation en fonction des conditions expérimentales. L'évaluation du devenir de ces cellules après leur implantation in vivo dans le cerveau de souris mimant des maladies neurodégénératives suggère un certain bénéfice thérapeutique, mais révèle également les difficultés et les risques (notamment tumoral) associés à ces stratégies. La résolution de ces obstacles est encore lointaine et, chez l'homme, s'ajoute une incertitude supplémentaire liée à notre maîtrise très balbutiante encore de la manipulation des cellules ES, et notamment de leur auto-renouvellement et de leur différenciation dans les lignages d'intérêt. <

\section{Cellules souches embryonnaires et thérapies cellulaires du système nerveux}

Anne-Catherine Fluckiger,

Colette Dehay, Pierre Savatier

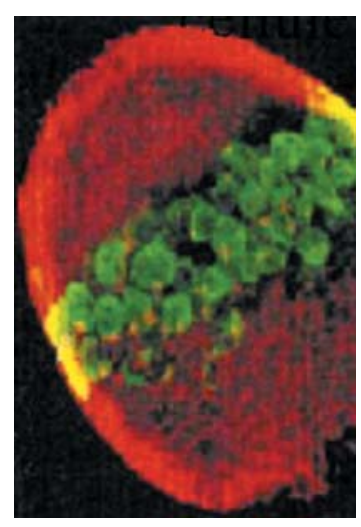

Les lignées de cellules souches embryonnaires de souris (cellules ES) sont isolées à partir des cellules de la
A.C. Fluckiger, C. Dehay: Inserm U.371, Cerveau et Vision, 18, avenue Doyen Lépine, 69500 Bron, France.

P. Savatier: Laboratoire de biologie moléculaire et cellulaire UMR 5665 , École Normale Supérieure de Lyon,

46, allée d'Italie,

69364 Lyon Cedex 07, France. pierre.savatier@ens-lyon.fr $\rightarrow) \mathrm{m} / \mathrm{s}$ 2002, $n^{\circ} 2$, p. 166 masse interne du blastocyste (Figure I) $(\rightarrow$ ). Elles sont pluripotentes, c'est-à-dire qu'une seule cellule $\varepsilon S$ est capable de produire tous les types cellulaires du fœtus Iorsqu'elle est replacée dans le blastocyste. In vitro, les cellules $\varepsilon S$ sont capables d'évoluer vers tous les lignages et de donner naissance à des descendants très différenciés comme des oligodendrocytes synthétisant la myéline [1], des cellules pancréatiques produisant de l'insuline [2] ou des cardiomyocytes contractiles [3]. Les cellules ES sont également immortelles: placées dans des conditions appropriées, elles s'autorenouvellent de façon illimitée, sans modification de leur caryotype. Le facteur clé de cet autorenouvellement est le LIF (leukemia inhibitory factor), qui active une voie de signalisation inhibant la différenciation $[4,5]$. Des lignées de cellules souches embryonnaires pluripotentes ont été isolées chez les primates humains $[6,7]$ et non humains (singes Rhésus et Cynomolgus) [8, 9]. Les cellules ES humaines, appelées $h E S$, présentent les mêmes propriétés cardinales que les cellules $\varepsilon S$ de souris (c'est-à-dire 
une immortalité et une pluripotence in vitro), mais en diffèrent par certaines caractéristiques phénotypiques et fonctionnelles. En particulier, les cellules hहS ne semblent pas dépendantes du LIF pour leur auto-renouvellement [4].

Parce qu'elles représentent une réserve cellulaire quasi illimitée et possèdent la capacité de se différencier en cellules spécialisées in vitro, les cellules ES constituent un outil de choix pour le développement de nouvelles approches thérapeutiques fondées sur le remplacement cellulaire. Les cellules hहS suscitent donc un intérêt grandissant au sein de la communauté scientifique et médicale, en même temps qu'un débat éthique lié à leur origine embryonnaire. La différenciation des cellules ES de souris et des cellules hES en cellules du système nerveux, puis leur implantation dans le cerveau de l'animal, ont fait l'objet de nombreux travaux parmi ceux les plus avancés dans le domaine des cellules souches thérapeutiques. Nous faisons le point sur l'état de ces recherches, puis nous décrivons les obstacles et évaluons les risques associés au développement de cette technologie pour le traitement des maladies du système nerveux.

\section{Les cellules \&S se différencient en neurones, en astrocytes et en oligodendrocytes in vitro}

\section{La différenciation} neuro-épithéliale des cellules ES de souris Les cellules ES se différencient spontanément en cellules exprimant des marqueurs du neuro-épithélium lorsqu'elles sont privées des facteurs permettant leur auto-renouvellement (LIF ou cellules nourricières). On a proposé l'hypothèse selon laquelle la différenciation dans la voie neurale serait adoptée par défaut, alors que la différenciation dans les autres lignages (ceux du mésoderme et de l'endoderme) exigerait l'action d'inducteurs spécifiques [10]. Cette propension à la différenciation en cellules neuro-épithéliales pourrait également résulter de l'action autocrine du facteur FGF4 (fibroblast growth factor 4), qui est un inducteur de la différenciation neurale naturellement exprimé dans les cellules $\varepsilon S$ de souris [11].

Plusieurs stratégies visant à orienter spécifiquement la différenciation des cellules ES en précurseurs neuraux (c'està-dire en cellules souches multipotentes à l'origine des neurones et des cellules gliales - astrocytes et oligodendrocytes) ont été élaborées. Pour la plupart, ces stratégies passent par une étape transitoire, le corps embryoïde, composé de cellules précurseurs représentatives des trois feuillets embryonnaires, ectoderme, mésoderme et endoderme (Figure 2). À partir des corps embryoïdes, deux pro-

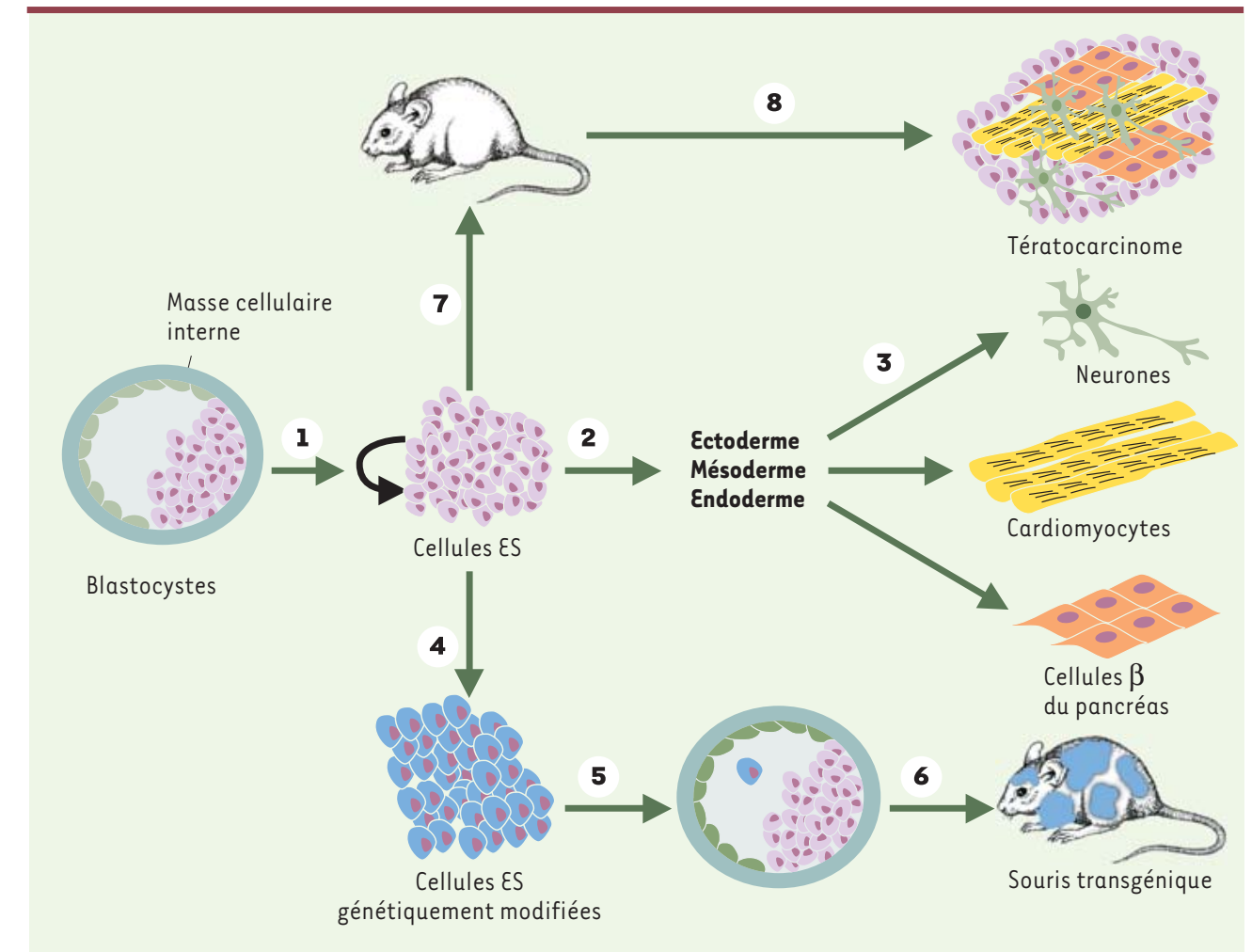

Figure 1. Origine et propriétés cardinales des cellules $\varepsilon S$. Les lignées de cellules $\varepsilon S$ sont fabriquées à partir de la masse cellulaire interne du blastocyste. I In vitro, les cellules ES sont capables de s'autorenouveler indéfiniment. Chez la souris, cet autorenouvellement est sous le contrôle du LIF (leukemia inhibitory factor). 2 In vitro, les cellules $\varepsilon S$ sont capables de se différencier en cellules des trois feuillets primitifs, ectoderme, mésoderme et endoderme. 3 Ces cellules précurseurs sont ensuite capables de se différencier en cellules matures fonctionnelles comme des neurones, des cardiomyocytes ou des cellules $\beta$ pancréatiques. 4 Les cellules $\varepsilon S$ peuvent être modifiées génétiquement. 5 Une seule cellule ainsi modifiée peut être ensuite implantée dans un blastocyste receveur où elle va poursuivre son programme de différenciation (6) et contribuer à la formation de tous les lignages constituant l'organisme adulte. 7 La greffe de cellules $\varepsilon S$ dans une souris adulte produit un tératocarcinome (8) composé de cellules souches embryonnaires pluripotentes et de cellules différenciées représentant les trois feuillets embryonnaires, ectoderme, mésoderme et endoderme. 
tocoles de référence ont été établis, l'un utilisant l'acide rétinoïque [12-14], l'autre fondé sur l'utilisation d'un milieu de culture dépourvu de sérum et enrichi en facteurs de croissance spécifiques [15]. Ces protocoles permettent d'obtenir de $30 \%$ [14] à $90 \%$ [15] de cellules présentant des caractéristiques de cellules neuro-épithéliales indifférenciées, caractérisées en particulier par l'expression du neurofilament nestine. Lorsqu'elles sont injectées dans les ventricules cérébraux de fœtus de rats, ces cellules neuro-

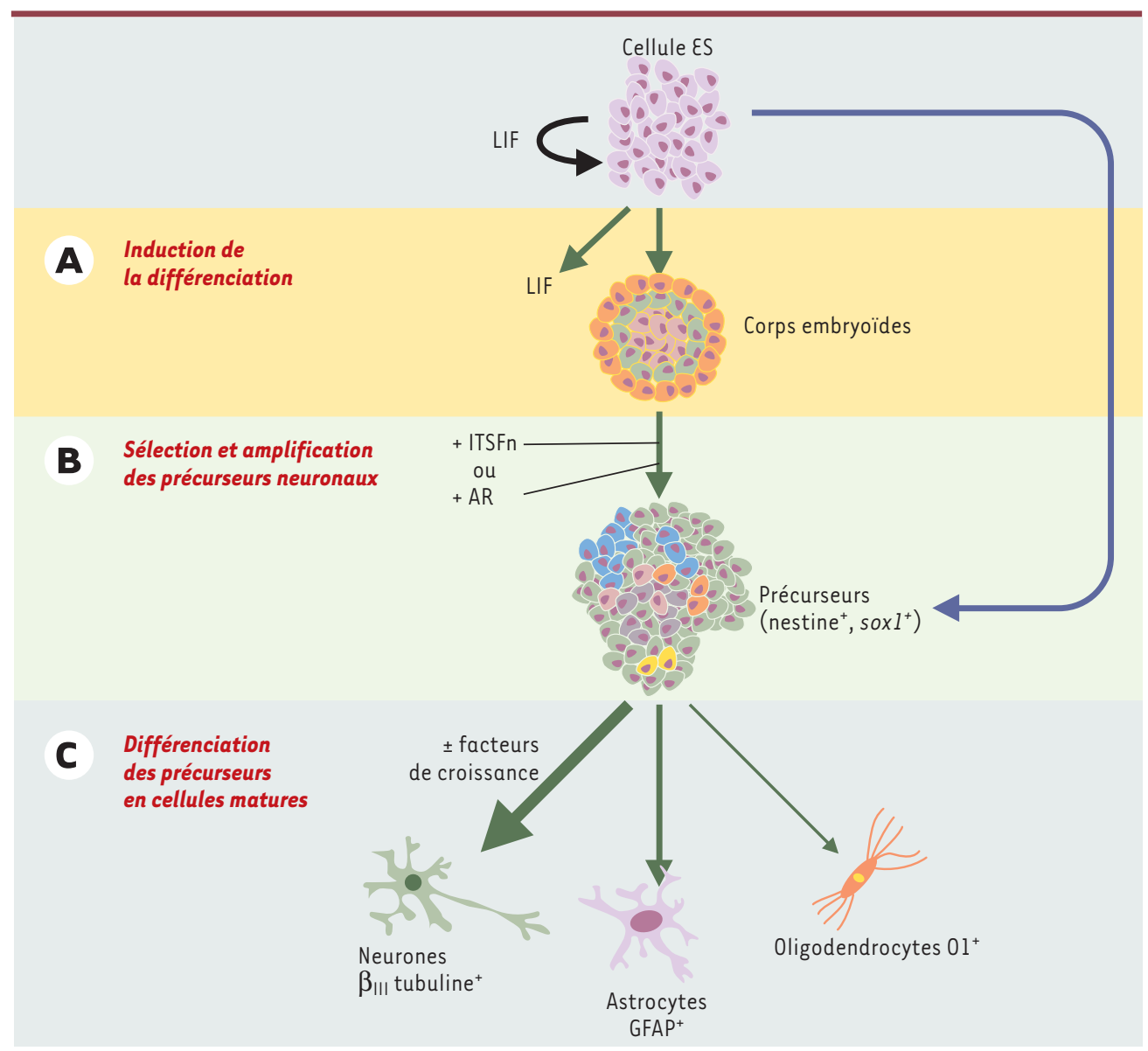

Figure 2. Principales étapes de la différenciation des cellules $\varepsilon S$ de souris en cellules du système nerveux. A. La différenciation est d'abord induite par la formation de corps embryoïdes. Ceux-ci sont obtenus en cultivant les cellules $\varepsilon S$ en suspension pendant 4 à 7 jours. Les corps embryoïdes sont constitués d'une mosaïque de cellules engagées dans la voie de l'ectoderme, du mésoderme et de l'endoderme. $\boldsymbol{B}$. Dans un deuxième temps, la différenciation dans la voie neuro-épithéliale est stimulée en cultivant les corps embryoïdes dans un milieu composé d'insuline, de transferrine, de sélénite de sodium, de fibronectine (ITSFn) pendant 7 jours, ou en présence d'acide rétinoïque (AR) pendant 4 jours. Il en résulte la formation d'une population de cellules neuro-épithéliales majoritairement caractérisées par l'expression du neurofilament nestine ou du gène pan-neural sox-1. Ces cellules présentent des caractéristiques génétiques de précurseurs neuronaux, c'est-à-dire de cellules multipotentes capables de se différencier en cellules neuronales et gliales (astrocytes et oligodendrocytes). C. La différenciation terminale est induite par la privation, ou au contraire l'ajout de facteurs de croissance ou d'hormones. Cette différenciation terminale résulte en la production de neurones exprimant le marqueur $\beta_{\mid 1}$-tubuline, d'astrocytes exprimant le marqueur GFAP (glial fibrillary acidic protein), et d'oligodendrocytes exprimant le marqueur 01 . Les proportions relatives de ces trois catégories cellulaires dépendent de la nature des facteurs ajoutés. La voie des corps embryoïdes peut, dans certaines conditions, être court-circuitée (flèche bleue en haut à droite). épithéliales se différencient spontanément en neurones, astrocytes et oligodendrocytes. Les neurones issus des cellules greffées émettent des réseaux axonaux de densité et d'orientation similaires à celles des faisceaux axonaux endogènes. Les astrocytes et les oligodendrocytes ont également une morphologie et une distribution spatiale comparables à celles des cellules de l'hôte. Les cellules neuro-épithéliales dérivées des cellules ES sont donc capables de contribuer au développement du système nerveux central, révélant ainsi un fort potentiel de différenciation neuronale et gliale caractéristique des précurseurs neuronaux [16].

Des populations de cellules exprimant majoritairement (> 90\%) des marqueurs neuro-épithéliaux comme le neurofilament nestine ou le gène panneural sox-1 peuvent être également obtenues, sans passer par l'étape intermédiaire du corps embryoïde, sous l'effet de milieux de culture définis [11] ou conditionnés [17], ou grâce à l'expression du gène «proneural » Sfrp2 codant pour la secreted frizzledrelated protein-2 [18]. Le facteur Sfrp2 semble stimuler la différenciation des cellules ES dans la voie neurale en inhibant la signalisation dépendante des facteurs Wnt impliquée dans la différenciation mésodermique. Aucune étude in vivo n'est venue, pour l'instant, confirmer le potentiel de différenciation neuronale et gliale des cellules neuro-épithéliales ainsi produites.

\section{La différenciation} neuronale et gliale des cellules ES de souris In vitro, les précurseurs neuro-épitheliaux dérivés des cellules ES sont capables de se différencier 
et de former des populations cellulaires mixtes composées en majorité de neurones (60-80\%), d'astrocytes (10-15\%) et d'oligodendrocytes (environ $3 \%$ ). Les neurones ainsi obtenus possèdent des canaux ioniques $\left(\mathrm{K}^{+}\right.$, $\mathrm{Ca}^{2+}$ et $\mathrm{Na}^{+}$) dépendants du voltage. Ils sont également capables de former des synapses GABAergiques et glutamatergiques et de transmettre des potentiels d'action post-synaptiques, indiquant une maturation fonctionnelle avancée $[13,15]$. Une grande diversité de soustypes neuronaux est observée, avec cependant une prédominance des neurones à glutamate et à GABA. Les neurones à dopamine ou à sérotonine sont obtenus avec une fréquence beaucoup plus faible (< 1\%) [15]. L'acide rétinoïque favorise la différenciation en neurones cholinergiques et glycinergiques [19].

L'enrichissement des populations de cellules différenciées en sous-types cellulaires spécifiques constitue une étape clé dans la perspective d'utilisation des cellules ES en thérapie cellulaire. Plusieurs variantes des protocoles de différenciation décrits précédemment ont été proposées afin d'augmenter la représentativité de certains types cellulaires dans la population finale. Par exemple, le facteur Sonic hedgehog (Shh), en association avec l'acide rétinoïque, oriente la différenciation en précurseurs des motoneurones spinaux cholinergiques (20-30\%) [20]. Au contraire, le facteur de croissance PDGF (platelet-derived growth factor) et l'hormone thyroïdienne favorisent la différenciation en oligodendrocytes (20-30\%) [1, 21, 22]. Cultivés en présence de neurones, ces oligodendrocytes sont capables de myéliniser les axones, indiquant un important degré de maturité fonctionnelle [21]. La différenciation des cellules ES en neurones dopaminergiques mésencéphaliques - neurones de la substance noire dont la dégénérescence caractérise la maladie de Parkinson - a fait l'objet d'études approfondies. Ainsi le facteur Shh, en synergie avec le facteur de croissance FGF8, stimule la différenciation en neurones dopaminergiques (34\%), mais aussi en neurones sérotoninergiques (23\%) [23]. L'efficacité de ce protocole de différenciation est significativement augmentée par la surexpression du gène nurr-1 [24]. Ce gène code pour un facteur de transcription nuclear receptor related-1 (Nurr-1) qui, in vivo, joue un rôle déterminant dans la différenciation des précurseurs mésencéphaliques en neurones dopaminergiques. En outre, Nurr-1 règle la transcription du gène de la tyrosine hydroxylase, l'enzyme limitante de la synthèse de la dopamine. La surexpression du gène nurr-1 exerce un effet synergique sur la différenciation dopaminergique des précurseurs neuronaux induite par FGF8 et Shh. Ce protocole est actuellement le plus performant pour produire des neurones dopaminergiques à partir de cellules ES de souris. Soixante-dix-huit pour cent des neurones ainsi produits expriment les marqueurs des neurones dopaminergiques caractérisés par une forte production de dopamine.

Malgré la multiplication des « recettes », les populations cellulaires différenciées, qu'elles soient de phénotype neuronal ou glial, ne sont pas homogènes [25]. L'hétérogénéité de la population de précurseurs neuroépithéliaux dont elles sont issues pourrait l'expliquer: bien que ces précurseurs expriment tous des marqueurs du neuro-épithélium, seule une sous-population serait constituée de véritables précurseurs neuronaux multipotents capables, sous l'action d'inducteurs spécifiques, de se différencier dans tous les sous-types neuronaux et gliaux. La maîtrise de l'identité des précurseurs pourrait donc se révéler un élément déterminant dans le contrôle de la différenciation en sous-types neuronaux d'intérêt thérapeutique.

\section{La différenciation neuronale et \\ gliale des cellules heS}

Comme dans le cas des cellules ES de souris, la formation des corps embryoïdes ou l'épuisement du milieu de culture provoque la différenciation spontanée des cellules hES en cellules exprimant majoritairement des marqueurs de cellules neuro-épithéliales. Ces cellules peuvent être alors amplifiées en présence des facteurs FGF2 et EGF (epidermal growth factor), et former des amas exprimant le neurofilament nestine et la molécule d'adhérence $\mathrm{N}$ CAM (neural cell adhesion molecule), amas semblables aux neurosphères produites dans des cultures primaires de cellules souches neurales de cerveau adulte [26, 27]. La culture de ces neurosphères sur un substrat d'adhérence induit une différenciation terminale majoritairement en neurones et en astrocytes, la différenciation en oligodendrocytes restant marginale [26]. L'induction de la différenciation neuronale par l'acide rétinoïque permet l'obtention de populations mixtes de neurones GABAergiques, dopaminergiques, glycinergiques et glutamatergiques caractérisés par des critères immunohistochimiques, ainsi que d'astrocytes. Les neurones ainsi obtenus ont un degré de maturation fonctionnelle significatif. Des analyses en imagerie calcique (visualisation de la réponse calcique) ont mis en évidence la réponse de ces neurones à divers neurotransmetteurs (GABA, acétylcholine). L'analyse des propriétés de membranes montre que les neurones ainsi obtenus expriment des canaux sodiques et potassiques dépendants du voltage et sont capables d'engendrer des potentiels d'action [28]. Les cellules hहS semblent donc capables de se différencier in vitro en neurones exprimant des caractéristiques fondamentales des neurones matures. 
La maîtrise de la différenciation des cellules hहS en sous-types neuronaux spécifiques en est à ses balbutiements. Des cellules ES de macaque Cynomolgus ont pu être induites à se différencier en neurones dopaminergiques avec une efficacité de $35 \%$, grâce à un procédé de co-culture avec une lignée d'origine stromale. Les neurones ainsi produits expriment les caractéristiques cardinales des neurones dopaminergiques mésencéphaliques [29]. Il est vraisemblable que ce procédé permettra également d'induire la différenciation des cellules hहS dans le lignage dopaminergique.

\section{Les cellules différenciées in vitro à partir des cellules $\varepsilon S$ survivent et fonctionnent après transplantation chez l'animal}

La capacité des cellules, produites in vitro à partir de cellules ES, de coloniser des tissus hôtes après transplantation puis à restaurer une fonction biologique défectueuse, a été évaluée récemment dans le contexte de trois approches thérapeutiques du système nerveux (Figure 3): la réinnervation de cibles musculaires par les motoneurones spinaux, la remyélinisa-

$\rightarrow) m / s \quad$ tion axonale par les oligodendrocytes et 2001, $n^{\circ} 10$, la restauration de la neurotransmission p. 1089 dopaminergique striato-corticale $(\rightarrow)$.
Quatre stratégies de greffe ont été évaluées; trois reposent sur la transplantation de cellules immatures dont le devenir est dicté par l'environnement de l'hôte: greffe de cellules de corps embryoïdes, greffe de précurseurs neuronaux multipotents et greffe de précurseurs gliaux (précurseurs des astrocytes et des oligodendrocytes) bipotents; la quatrième stratégie fait appel à la greffe de neurones post-mitotiques engagés dans la voie de différenciation correspondant au phénotype d'intérêt.

\section{Greffes de motoneurones spinaux et}

formation de synapses neuromusculaires

Des précurseurs de motoneurones dérivés des cellules \&S de souris ont été injectés dans la moelle épinière d'embryons de poulet afin d'explorer dans quelle mesure, après différenciation terminale, ils pouvaient s'intégrer et innerver les cibles musculaires appropriées [20]. Les motoneurones dérivés de ces précurseurs adoptent une localisation ventrolatérale typique des motoneurones endogènes et émettent des axones poussant dans la corne ventrale et empruntant des trajets périphériques identiques à ceux des axones des motoneurones endogènes. Lors de leur arrivée sur leur cible musculaire, les axones des motoneurones dérivés des cellules ES développent une arborisation fournie et montrent des indices

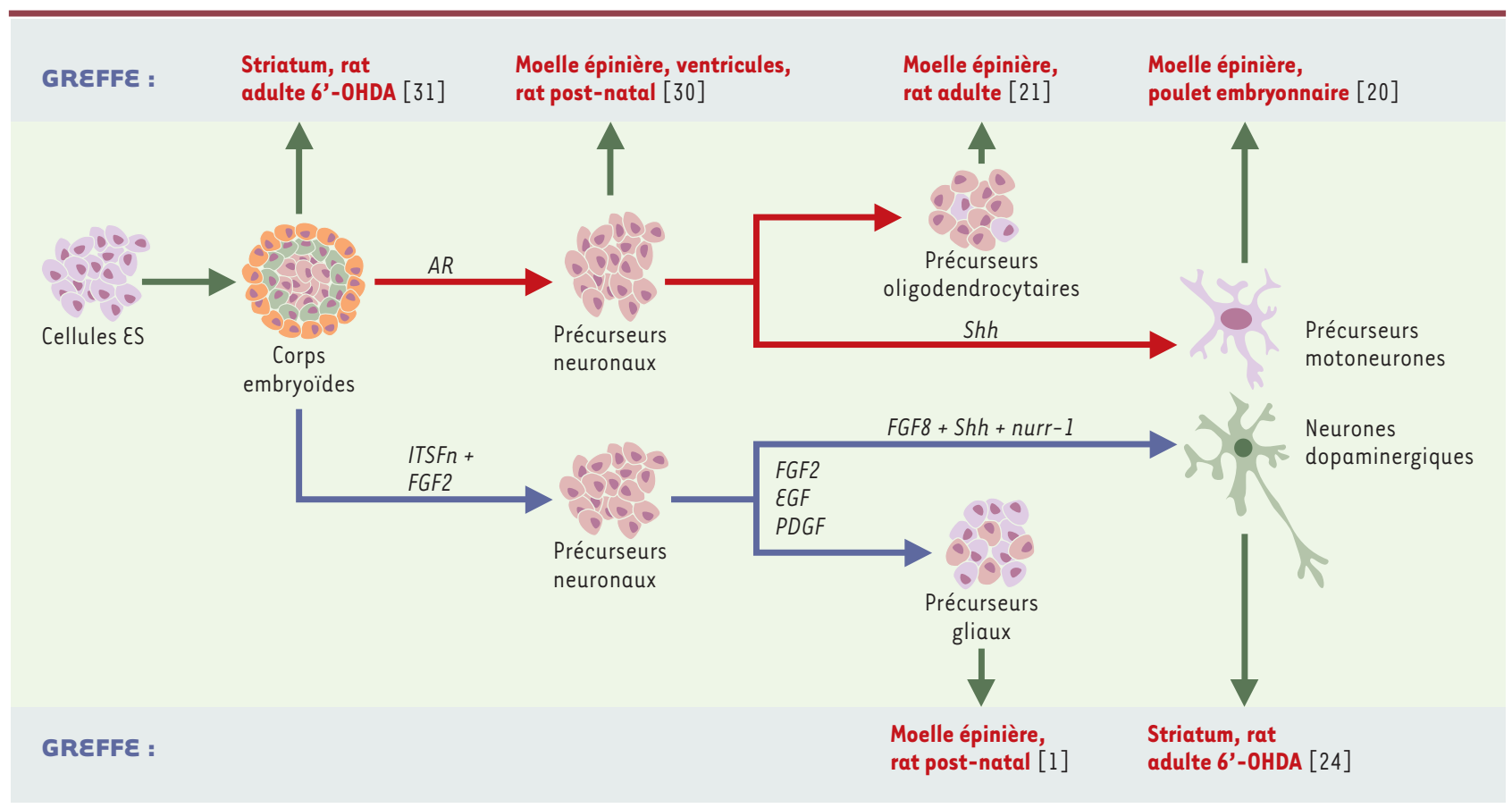

Figure 3. Greffes de cellules obtenues par différenciation in vitro des cellules ES de souris (selon les protocoles décrits dans la Figure 2). Les greffes ont été réalisées avec des cellules de corps embryoïdes [31], des précurseurs neuronaux multipotents [30], des précurseurs gliaux [1], des précurseurs oligodendrocytaires [21], des pércurseurs neuronaux [20], ou des neurones post-mitotiques [24]. ITSFn: insuline + transferrine + sélénite + fibronectine; AR: acide rétinoïque; Shh: Sonic hedgehog; FGF2: fibroblast growth factor 2; દGF: epidermal growth factor; PDGF: plateletderived growth factor; nurr-1: nuclear receptor related-1. 
de différenciation pré-synaptique, suggérant la possibilité d'établissement de jonctions neuro-musculaires fonctionnelles.

Myélinisation des neurones spinaux chez le rat après greffe de précurseurs gliaux dérivés de cellules $\varepsilon S$ Des précurseurs neuronaux dérivés des cellules ES de souris ont été injectés chez des rats adultes dont la moelle épinière a été préalablement lésée [30]. Cinq semaines après l'injection, les cellules transplantées ont rempli l'espace lésé et se sont différenciées en oligodendrocytes ( $43 \%$ ), en astrocytes ( $19 \%$ ) et en neurones $(8 \%)$. Les rats ainsi traités montrent une récupération fonctionnelle partielle dont le substrat cellulaire reste à élucider. La rapidité de la récupération fonctionnelle associée à une différenciation majoritairement orientée vers la voie oligodendrocytaire conduit les auteurs à avancer l'hypothèse d'une augmentation de conductance dans les axones non lésés, qui résulterait d'une stimulation de la myélinisation.

Afin d'évaluer la capacité des cellules greffées de myéliniser les axones de l'hôte, des précurseurs gliaux produits à partir de cellules ES de souris ont été injectés dans la moelle épinière de jeunes rats $m$ d (myelin deficien) [1]. Deux semaines après l'injection, les cellules greffées se sont différenciées en oligodendrocytes capables de myéliniser les axones de l'hôte à la périphérie du site d'injection. Des expériences similaires ont été réalisées avec des précurseurs oligodendrocytaires issus de cellules $E S$ et injectés dans le segment thoracique de moelle épinière de souris shiverer déficientes en myelin basic protein [21]. Deux semaines après l'injection, les auteurs rapportent une répartition des oligodendrocytes greffés comparable à celle qui est observée chez les animaux normaux, accompagnée d'un processus de myélinisation des axones de l'hôte à la périphérie du site d'injection. Ces résultats démontrent la capacité de précurseurs neurogliaux produits in vitro à partir des cellules ES de souris de se différencier en oligodendrocytes capables de régénérer la myéline in vivo. Cependant, on ne dispose d'aucune information sur la persistance de cette régénération dans les mois qui suivent l'injection.

\section{Restauration de la neurotransmission}

\section{striatocorticale dans un modèle de rat}

\section{de la maladie de Parkinson}

Chez le rongeur, l'administration de 6'-hydroxy dopamine (6'-OHDA) détruit les neurones dopaminergiques de la substance noire et leurs projections striatales, mimant la dégénérescence neuronale observée dans la maladie de Parkinson. Des neurones dopaminergiques mésencéphaliques (neurones $\mathrm{TH}^{+}$) produits in vitro à partir de cellules ES de souris ont été greffés dans le striatum de rat trois jours après une lésion induite par la 6'-OHDA [24]. Les analyses électrophysiologiques réalisées 2 à 5 mois après la greffe montrent la capacité des neurones $\mathrm{TH}^{+}$ayant survécu ( $5 \%$ ) de produire des potentiels d'action de durée et de fréquence identiques à celles des neurones $\mathrm{TH}^{+}$de l'hôte, ainsi qu'à induire des potentiels post-synaptiques inhibiteurs caractéristiques des neurones à dopamine. Par ailleurs, la stimulation extracellulaire de neurones du greffon induit des potentiels post-synaptiques excitateurs aussi bien dans les neurones adjacents a greffon que dans les neurones de l'hôte. Les neurones $\mathrm{TH}^{+}$, dérivés des cellules $\varepsilon S$, sont donc capables d'établir des synapses fonctionnelles avec les neurones de l'hôte et possèdent les caractéristiques électrophysiologiques de neurones dopaminergiques mésencéphaliques.

Les rats traités par la 6'-OHDA présentent également des déficits moteurs spontanés analogues à ceux qui sont observés dans la maladie de Parkinson. Le bénéfice fonctionnel de ces greffes a été évalué grâce une batterie de tests comportementaux tels que la fréquence d'utilisation de la patte antérieure ipsilatérale à la lésion striatale pour la réception après un saut ou pour l'obtention de nourriture. Chez les rats greffés avec des neurones $\mathrm{TH}^{+}$, on observe une amélioration significative de la fréquence d'utilisation de la patte ipsilatérale pour l'exécution de ces tâches, démontrant ainsi que les neurones dérivés des cellules ES sont capables de restaurer une fonction et de participer à l'exécution de tâches intégratives [24].

Des résultats similaires ont été obtenus avec la transplantation de cellules de corps embryoïdes (cellules « naïves ») injectées dans le striatum lésé par la 6'OHDA [31]. Environ 4 mois après la greffe, une fraction des cellules injectées se sont différenciées spontanément en neurones exprimant des marqueurs dopaminergiques mésencéphaliques. Les résultats d'IRM (imagerie par résonance magnétique) fonctionnelle révèlent une augmentation d'activité dans le striatum greffé et le cortex sensorimoteur après administration d'amphétamines chez les animaux greffés, indiquant un relargage de dopamine dans la boucle corticostriatale. Ces observations témoignent d'une intégration fonctionnelle des cellules greffées conduisant à un certain niveau de restauration de la neurotransmission striatocorticale.

Il est important de noter que les analyses électrophysiologiques, fonctionnelles et comportementales, ont été réalisées, selon les cas, 2 à 12 semaines après les greffes. On ne dispose d'aucune information au-delà de trois mois. Contrairement aux greffes de cellules différenciées, l'innocuité de la transplantation de cellules « naïves » 
n'est pas atteinte, dans la mesure où $20 \%$ des rats ayant reçu une greffe de cellules «naïves » ont développé un tératome au niveau du site d'injection [31].

\section{De la démonstration expérimentale à l'application clinique}

\section{Maîtriser la différenciation des cellules ES}

L'utilisation des cellules ES en thérapie cellulaire implique la maîtrise de leur différenciation vers des lignages cellulaires spécifiques. Dans cette perspective, le développement de méthodes permettant d'orienter la différenciation vers les lignages désirés, puis d'assurer la maturation en cellules fonctionnelles, représente un enjeu majeur. La différenciation neuronale dopaminergique des cellules $\varepsilon S$, étudiée par de nombreuses équipes, apparaît comme un paradigme expérimental: les actions conjointes de dix facteurs de croissance ou d'attachement, ajoutées à celle du facteur de transcription pro-dopaminergique Nurr-1, sont nécessaires pour promouvoir la différenciation des cellules ES de souris en neurones dopaminergiques mésencéphaliques avec une efficacité proche de $80 \%[23,24]$. Sans inducteur spécifique, seules 0,1 à $1 \%$ des cellules $E S$ adoptent spontanément ce phénotype. Pour d'autres sous-types neuronaux d'intérêt clinique comme les neurones GABAergiques du striatum ou les neurones cholinergiques de l'hippocampe, ou d'autres catégories cellulaires comme les cellules $\beta$ pancréatiques, la maîtrise de la différenciation in vitro en est à ses balbutiements. L'expression ectopique de gènes clés de la différenciation pourra se révéler indispensable afin d'orienter efficacement le devenir de la cellule et de spécifier au mieux son identité finale. L'utilisation d'un système d'expression génique inductible représente un outil puissant permettant d'activer l'expression des gènes clés au moment opportun et d'éviter ainsi les effets délétères résultant de l'expression non contrôlée de ces gènes lors des étapes très précoces de la différenciation $[32,33]$.

La greffe de précurseurs neuronaux multipotents dérivés des cellules ES constitue une voie alternative à la greffe de cellules matures pour la réparation tissulaire. Les expériences réalisées chez la souris démontrent la capacité de ces précurseurs, dérivés des cellules ES humaines ou murines, de se différencier en neurones et en oligodendrocytes matures après greffe chez l'animal. Cette différenciation in situ témoigne donc de la remarquable capacité de ces précurseurs de répondre à des signaux environnementaux locaux. Là aussi, l'orientation de la différenciation vers un lignage spécifique reste un enjeu majeur. Les populations de précurseurs sont générale- ment hétérogènes, constituées de précurseurs de nature et de maturité différentes. Après transplantation, ces précurseurs multipotents se différencient de façon incontrôlée en de nombreux types cellulaires différents. II sera donc nécessaire de développer des stratégies permettant, d'une part, de maîtriser totalement les caractéristiques des précurseurs multipotents engendrés in vitro et, d'autre part, de contrôler étroitement leur devenir après implantation. On peut envisager d'introduire des mutations les empêchant de se différencier dans les lignages non désirés ou, au contraire, d'activer au moment de la transplantation l'expression de gènes maîtres capables de diriger leur différenciation dans le lignage d'intérêt.

À l'heure actuelle, aucune méthode d'induction de la différenciation des cellules $\varepsilon S$ ne permet d'obtenir une population pure d'un type cellulaire spécifique. Une stratégie de sélection génétique peut alors être mise en œuvre pour éliminer les cellules s'étant différenciées dans les autres lignages. Cette stratégie a été employée avec succès pour enrichir les cellules différenciées en précurseurs neuronaux, en cardiomyocytes ou en cellules $\beta$ pancréatiques, en utilisant les éléments de régulation des gènes codant respectivement pour le facteur de transcription pan-neural Sox-2, la myosine ou l'insuline $[2,3,22,34](\rightarrow)$. Cette méthode d'enrichissement apparaît comme une force d'appoint permettant, in $(\rightarrow) \mathrm{m} / \mathrm{s}$

2001, $n^{\circ} 10$, p. 1088 dont la différenciation aura été préalablement privilégiée grâce à l'action des facteurs de croissance et des facteurs de transcription.

\section{Transférer la technologie aux cellules hES}

Si des avancées spectaculaires ont été réalisées ces dernières années dans la manipulation des cellules ES de souris, de nombreux progrès restent à accomplir dans la maîtrise de l'auto-renouvellement et de la différenciation in vitro des cellules hES. L'amplification des cellules hES est encore très délicate, les conditions de leur autorenouvellement et de leur croissance clonale sont imparfaitement maîtrisées, rendant leur modification génétique plus difficile que dans le cas des cellules ES de souris [35]. L'identification des facteurs et des mécanismes contrôlant l'auto-renouvellement des cellules hES constitue donc un préalable incontournable pour envisager d'utiliser ces cellules en thérapie cellulaire. De nouvelles conditions de culture facilitant l'autorenouvellement des cellules hहS doivent donc être mises au point, avec la contrainte impérative de s'affranchir de l'utilisation de cellules nourricières ou de milieux conditionnés produits à partir d'autres espèces afin 
d'éliminer le risque de transmission interspécifique d'agents pathogènes. Des progrès doivent également être réalisés dans la maîtrise de la différenciation des cellules hहS. En effet, la réponse de ces cellules à certains facteurs de croissance diffère de celle des cellules \&S de souris, soulignant la nécessité de développer de nouvelles méthodes de différenciation spécifiques $[26,27]$.

\section{Démontrer l'efficacité et l'innocuité de la transplantation cellulaire chez le primate non humain}

Tous les travaux examinant les résultats de greffes de cellules dérivées de cellules $\varepsilon S$ ont été réalisés chez des rongeurs. Si ces expériences ont apporté la preuve du bien-fondé du concept de réparation tissulaire fondée sur la technologie des cellules ES, un nombre croissant de travaux met en évidence des différences fondamentales dans le développement et l'organisation du système nerveux central entre le rongeur et le primate, limitant ainsi les extrapolations qui peuvent être faites entre les deux espèces [36]. Il est donc impératif, avant de pouvoir envisager tout test clinique, de procéder à l'évaluation de l'efficacité et de l'innocuité des cellules dérivées de cellules $\varepsilon S$ en recherche préclinique dans un modèle de primate non humain. Le primate non humain - singe Rhésus -, du fait de la similitude de l'organisation de ses structures corticales et sous-corticales avec celle du cerveau humain, constitue le modèle privilégié pour les prédictions en neurologie humaine, notamment en ce qui concerne les maladies neurodégénératives. Le singe Rhésus, pour lequel il existe un modèle fiable de la maladie de Parkinson [37], représente le modèle préclinique de choix pour étudier le bénéfice thérapeutique des greffes de cellules dérivées des cellules \&S. C'est en effet le seul modèle animal qui permettra une analyse fine de la récupération des déficits cognitifs associés à la maladie de Parkinson, déficits impossibles à tester chez les rongeurs. Par ailleurs, cette espèce présente une durée de vie suffisamment longue pour étudier la survie des cellules greffées et examiner les consé-

$\rightarrow) \mathrm{m} / \mathrm{s}$ 2001, $n^{\circ} 10$, p. 1090

\section{quences à long terme des greffes [38] $(\rightarrow)$.}

Les cellules \&S forment des tératocarcinomes malins non métastatiques lorsqu'elles sont implantées dans un site ectopique hors du contexte embryonnaire. La possibilité que des cellules $E S$ résiduelles, contaminant la préparation de cellules différenciées, continuent à proliférer et éventuellement à former des tumeurs chez le receveur constitue un danger [31, 39]. Un tel danger n'a pas été rapporté dans les expériences de greffes utilisant des précurseurs neuronaux multipotents ou des cellules matures. II conviendra de déterminer si cela est égale- ment le cas chez le primate non humain, grâce à un suivi à long terme des animaux greffés. Une stratégie de sélection génétique négative pourra être mise en œuvre préventivement afin d'éliminer totalement les cellules ES non différenciées avant transplantation [39].

\section{Maîtriser le rejet}

Le rejet du greffon par le receveur est une cause d'échec importante lors des allotransplantations. Ce risque est diminué par le choix d'un donneur présentant la meilleure compatibilité possible avec le receveur et par un traitement immunodépresseur du receveur. Des stratégies consistant à modifier les gènes du système d'histocompatibilité du greffon ont été évoquées mais restent toutes insatisfaisantes pour l'instant. Quant à l'inactivation des gènes du complexe majeur d'histocompatibilité (CMH) chez la souris, elle n'a pas permis d'éviter le rejet [40]. Le remplacement des gènes du CMH des cellules hES d'origine par les gènes homologues $d u$ patient est difficilement envisageable dans les conditions technologiques actuelles. Enfin, le transfert nucléaire d'une cellule adulte de l'hôte dans un ovocyte a permis de dériver des cellules ES immunologiquement compatibles, mais avec une très faible efficacité (seulement 1 à $3 \%$ des noyaux transférés conduisent à une lignée de cellules \&S chez la souris) [41]. La transplantation cellulaire dans le système nerveux central constitue un cas particulier. Le cerveau ne possédant pas de tissus lymphoïdes, la réponse immune ne peut résulter que de l'infiltration de cellules immunitaires à partir des vaisseaux et des capillaires sanguins et lymphatiques. Des greffes de neurones fœtaux dans le striatum chez l'homme ont montré que les cellules transplantées survivent jusqu'à dix ans après la greffe, sans signe de lésion dégénérative du greffon [42, 43], indiquant une bonne tolérance du greffon fœtal par le système immunitaire. Il conviendra de déterminer si les cellules différenciées dérivées de cellules ES sont également bien tolérées et, dans ce contexte, la mise en œuvre d'allotransplantations de cellules différenciées dérivées de cellules ES chez le singe macaque est une étape importante pour assurer la validité et l'innocuité de cette approche avant de l'appliquer à l'être humain. $\diamond$

\section{SUMMARY}

Embryonic stem cells and cell replacement therapies in the nervous system

Embryonic stem (ES) cells are pluripotential cells derived from the pre-implantation embryo. They can proliferate indefinetely in vitro while retaining pluripotency. ES cells can also be made to differentiate into a large variety of cell types in vitro. This has paved the way to 
research aimed at using ES-derived cells for cell replacement therapies. Hence, mouse ES cells can efficiently differentiate into neural precursors which can further generate functional neurons, astrocytes, and oligodendrocytes. Methods have also been developed to coax mouse ES-derived neural stem cells to differentiate into either dopaminergic neurons or motoneurons. Mouse ESderived neural stem cells, or their fully differentiated progeny, have been shown to survive, integrate, and to some extent, function following transplantation within appropriate rodent host tissue. Research on human ES cells is still in its infancy. Considerable work has to be done: (1) to master growth and genetic manipulation of human ES cells; (2) to master their differentiation into specific cell types; and (3) to demonstrate that they can provide long term therapeutical benefits upon grafting into damaged tissues in humans. From the ethical point of view, the establishment of appropriate primate model will be an obligatory prerequisite to clinical trials based on $£ S$ cells derivatives grafting. $\diamond$

\section{RÉFÉRENCES}

1. Brustle 0, Jones KN, Learish $\mathrm{RD}$, et al. Embryonic stem cell-derived glial precursors: a source of myelinating transplants. Science 1999; 285: 754-6.

2. Soria B, Roche $\varepsilon$, Berna G, Leon-Quinto T, Reig JA, Martin F. Insulin-secreting cells derived from embryonic stem cells normalize glycemia in streptozotocin-induced diabetic mice. Diabetes 2000; 49: 157-62.

3. Klug MG, Soonpaa MH, Koh Gy, Field LJ. Genetically selected cardiomyocytes from differentiating embronic stem cells form stable intracardiac grafts. J Clin Invest 1996; 98 : 216-24.
4. Smith A. Embryo-derived stem cells: of mice and men. Annu Rev Cell Dev Biol 2001; 17 : 435-62.

5. Burdon T, Smith AG, Savatier P. Signalling, cell cycle and pluripotency in embryonic stem cells. Trends Cell Biol 2002; 12 : 432-8.

6. Thomson JA, Itskovitz-Eldor J, Shapiro SS, et al. Embryonic stem cell lines derived from human blastocysts. Science 1998; 282: 1145-7.

7. Reubinoff $B \varepsilon$, Pera MF, Fong Cy, Trounson A, Bongso A. Embryonic stem cell lines from human blastocysts: somatic differentiation in vitro. Nat Biotechnol 2000; 18: 399-404.
8. Thomson JA, Kalishman J, Golos TG, et al. Isolation of a primate embryonic stem cell line. Proc Natl Acad Sci USA 1995; 92 : 7844-8.

9. Suemori H, Tada T, Torii R, et al. Establishment of embryonic stem cell lines from Cynomolgus monkey blastocysts produced by IVF or ICSI. Dev Dyn 2001; 222: 273-9.

10. Tropepe V, Hitoshi S, Sirard C, Mak TW, Rossant J, van der Kooy D. Direct neural fate specification from embryonic stem cells: a primitive mammalian neural stem cell stage acquired through a default mechanism. Neuron 2001; 30: 65-78.

11. Ying $Q L$, Stavridis $M$, Griffiths D, Li M, Smith A. Conversion of embryonic stem cells into neuroectodermal precursors in adherent monoculture. Nat Biotechnol 2003; 21 : 183-6.

12. Bain G, Kitchens D, Yao M, Huettner JE, Gottlieb DI. Embryonic stem cells express neuronal properties in vitro. Dev Biol 1995; 168: 342-57.

13. Strubing $C$, Ahnert-Hilger G, Shan J, Wiedenmann B, Hescheler J, Wobus AM. Differentiation of pluripotent embryonic stem cells into the neuronal lineage in vitro gives rise to mature inhibitory and excitatory neurons. Mech Dev 1995; 53: 275-87.

14. Fraichard A, Chassande 0 , Bilbaut C, Dehay C, Savatier $P$, Samarut J. In vitro differentiation of $E S$ cells into glial cells and functional neurons. J Cell Sci 1995; 108: 3181-8.

15. Okabe S, Forsberg-Nilsson K, Spiro AC, Segal M, McKay RD. Development of neuronal precursor cells and functional postmitotic neurons from embryonic stem cells in vitro. Mech Dev 1996; 59: 89-102.

16. Brustle 0, Spiro AC, Karram K, Choudhary K, Okabe S, McKay RD. In vitro- generated neural precursors participate in mammalian brain development. Proc Natl Acad Sci USA 1997; 94 : 14809-14.

17. Rathjen J, Haines BP, Hudson KM, Nesci A, Dunn $S$, Rathjen PD. Directed differentiation of pluripotent cells to neural lineages: homogeneous formation and differentiation of a neurectoderm population. Development 2002; 129 : 2649-61.

18. Aubert J, Dunstan $H$, Chambers I, Smith A. Functional gene screening in embryonic stem cells implicates Wnt antagonism in neural differentiation. Nat Biotechnol 2002; 20 : 1240-5.

19. Renoncourt $y$, Carroll $P$, Filippi P, Arce V, Alonso S. Neurons derived in vitro from \&S cells express homeoproteins characteristic of motoneurons and interneurons. Mech Dev 1998; 79: 185-97.

20. Wichterle H, Lieberam I, Porter JA, Jessell TM. Directed differentiation of embryonic stem cells into motor neurons. Cell 2002; 110: 385-97.

21. Liu S, Qu Y, Stewart TJ, et al. Embryonic stem cells differentiate into oligodendrocytes and myelinate in culture and after spinal cord transplantation. Proc Natl Acad Sci USA 2000; 97 : 6126-31.

22. Billon $N$, Jolicoeur $C$, Ying QL, Smith A, Raff M. Normal timing of oligodendrocyte development from genetically engineered, lineage-selectable mouse ES cells. J Cell Sci 2002; 115: 3657-65.

23. Lee SH, Lumelsky N, Studer L, Auerbach JM, McKay RD. Efficient generation of midbrain and hindbrain neurons from mouse embryonic stem cells. Nat Biotechnol 2000; 18 : 675-9. 
24. Kim JH, Auerbach JM, Rodriguez-Gomez JA, et al. Dopamine neurons derived from embryonic stem cells function in an animal model of Parkinson's disease. Nature 2002; 418 : 50-6.

25. Stavridis MP, Smith AG. Neural differentiation of mouse embryonic stem cells. Biochem Soc Trans 2003; 31: 45-9.

26. Reubinoff $B E$, Itsykson $P$, Turetsky T, et al. Neural progenitors from human embryonic stem cells. Nat Biotechnol 2001; 19 : 1134-40.

27. Zhang SC, Wernig $M$, Duncan ID, Brustle 0, Thomson JA. In vitro differentiation of transplantable neural precursors from human embryonic stem cells. Nat Biotechnol 2001; 19: 1129-33.

28. Carpenter MR, Inokuma MS, Denham J, Mujtaba T, Chiu CP, Rao MS. Enrichment of neurons and neural precursors from human embryonic stem cells. Exp Neurol 2001; 172: 383-97.

29. Kawasaki H, Suemori H, Mizuseki K, et al. Generation of dopaminergic neurons and pigmented epithelia from primate $\varepsilon S$ cells by stromal cellderived inducing activity. Proc Natl Acad Sci USA 2002; 99: 1580-5.

30. McDonald JW, Liu XZ, Qu Y, et al. Transplanted embryonic stem cells survive, differentiate and promote recovery in injured rat spinal cord. Nat Med 1999; 5: 1410-2.

31. Bjorklund LM, SanchezPernaute R, Chung $\mathrm{S}$, et al. Embryonic stem cells develop into functional dopaminergic neurons after transplantation in a Parkinson rat model. Proc Natl Acad Sci USA 2002; 99: 2344-9.

32. Kyba M, Perlingeiro RC, Daley GQ. HoxB4 confers definitive lymphoidmyeloid engraftment potential on embryonic stem cell and yolk sac hematopoietic progenitors. Cell 2002; 109: 29-37.

33. Vallier L, Mancip J, Markossian S, et al. An efficient system for conditional gene expression in embryonic stem cells and in their in vitro and in vivo differentiated derivatives.
Proc Natl Acad Sci USA 2001; 98: 2467-72.

34. Li M, Pevny L, Lovell-Badge $R$, Smith A. Generation of purified neural precursors from embryonic stem cells by lineage selection. Curr Biol 1998; 8: 971-4.

35. Zwaka TP, Thomson JA. Homologous recombination in human embryonic stem cells. Nat Biotechnol 2003; 21: 319-21.

36. Kennedy H, Dehay $C$. Cortical specification of mice and men. Cereb Cortex 1993; 3: 171-86.

37. Andringa G, Vermeulen RJ, Drukarch B, Renier WO, Stoof JC, Cools AR. The validity of the pretreated, unilaterally MPTP-treated monkeys as a model of Parkinson's disease: a detailed behavioural analysis of the therapeutic and undesired effects of the D2 agonist quinpirole and the Dl agonist SKF 81297. Behav Pharmacol 1999; 10: 163-73.

38. Svendsen CN, Smith AG. New prospects for human stem-cell therapy in the nervous system. Trends Neurosci 1999; 22: 357-64.

39. Odorico JS, Kaufman DS, Thomson JA. Multilineage differentiation from human embryonic stem cell lines. Stem Cells 2001; 19 : 193-204.

40. Grusby MJ, Auchincloss H Jr, Lee $\mathrm{R}$, et al. Mice lacking major histocompatibility complex class I and class II molecules. Proc Natl Acad Sci USA 1993; 90 : 3913-7.

41. Munsie MJ, Michalska AE, O'Brien CM, Trounson AO, Pera MF, Mountford PS. Isolation of pluripotent embryonic stem cells from reprogrammed adult mouse somatic cell nuclei. Curr Biol 2000; 10 : 989-92.

42. Piccini P, Brooks DJ, Bjorklund A, et al. Dopamine release from nigral transplants visualized in vivo in a Parkinson's patient. Nat Neurosci 1999; 2: 1137-40.

43. Freeman TB, Cicchetti F, Hauser RA, et al. Transplanted fetal striatum in Huntington's disease: phenotypic development and lack of pathology. Proc Natl Acad Sci USA 2000; 97: 13877-82.

\section{TIRÉS À PART}

P. Savatier

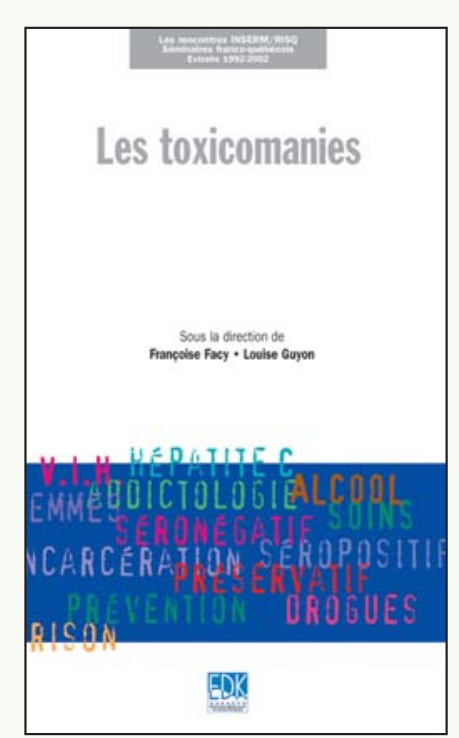

ISBN: 2-84254-080-8 296 pages

\section{Bon de commande}

À retourner à EDK, 10 Villa d'Orléans - 75014 PARIS

Tél.: 0153910606 - Fax: 0153910607 - E-mail: editorial@edk.fr

NOM:

Prénom:

Adresse:

Code postal:

Ville:

Pays:

Fonction:

Je souhaite recevoir l'ouvrage Les toxicomanies: $16 €+3 €$ de port $=19 €$ TTC

en ............... exemplaire, soit un total de ............................... $€$

$\square$ Par chèque, à l'ordre de $\mathbf{E} \mathbf{D} \mathbf{K}$

$\square$ Par carte bahcie: $\square$ Visa

$\square$ Eurocard/Mastercard

American Express

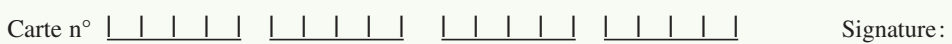

